The photograph that was used on the cover of the January issue of Nature Neuroscience was incorrectly credited. Dr. T. Self of the University of Nottingham kindly provided the image.

\title{
Modulation of cochlear hair cells by the auditory cortex in the mustached bat
}

Zhongju Xiao and Nobuo Suga

Nat. Neurosci. 5, 57-63 (2002)

Figure $1 \mathrm{~b}$ reproduced poorly. The correct figure appears below. We regret the error.

b

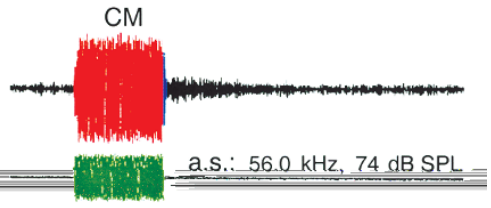

Fig. 1. Cortical over-representation of and sharply tuned hair cell responses to a $61 \mathrm{kHz}$ sound. (a) The primary auditory cortex. The dashed lines and numbers indicate iso-best frequency lines. The Doppler-shifted constant frequency (DSCF) area (pink) over-represents a sound between 60.6 and $62.3 \mathrm{kHz}$. mca, middle cerebral artery. (b, c) Cochlear microphonic responses (CMs) evoked by 2.0-ms tone bursts. Frequency and amplitude of an acoustic stimulus (a.s.) are indicated in $\mathrm{kHz}$ and $\mathrm{dB} S \mathrm{SP}$, respectively. The $\mathrm{CM}$ and $\mathrm{CM}$-after are indicated in red and blue, respectively. The data in (b) and (c) were obtained from a single cochlea. 